\title{
Validación de un cuestionario para la valoración de la actividad física en escolares adolescentes Validation of a questionnaire assessing school physical activity in adolescents
}

\author{
Rossana Gómez Campos ${ }^{1}$, Élmer Vilcazán², Miguel De Arruda ${ }^{1}$, \\ Jeffersson E Hespañol ${ }^{3}$, Marco Antonio Cossio-Bolaños ${ }^{1}$ \\ ${ }^{1}$ Universidad Estadual de Campinas, UNICAMP, Brasil. \\ ${ }^{2}$ Instituto Superior Pedagógico Arequipa, Perú. \\ ${ }^{3}$ Pontificia Universidad Católica de Campinas, SP, Brasil.
}

\begin{abstract}
Resumen
Introducción: En el Perú, hasta el momento no existe un cuestionario que pueda ser utilizado para valorar el nivel de actividad física relacionada a la salud de niños y adolescentes, así como informaciones sobre medidas de reproducibilidad. Objetivo: Proponer y validar un cuestionario de actividad física para escolares adolescentes que habitan en altitud moderada. Diseño: Estudio descriptivo simple. Institución: Facultad de Educación Física, UNICAMP, Brasil. Participantes: Escolares de 10 a 18 años. Intervenciones: La muestra fue compuesta por 116 alumnos, seleccionados de forma probabilística estratificada, siendo 58 varones y 59 damas, que constituyeron el 20\% de la población de escolares del Colegio Nacional Inmaculada Concepción, localizado en el Distrito de José Luis Bustamante y Rivero de Arequipa (Perú). El cuestionario contó con 11 preguntas que valoraron el nivel de actividad física por medio de cuatro indicadores, como tipo de actividad, frecuencia, duración e intensidad. Para la determinación de la reproducibilidad, se utilizó el procedimiento de test y re test, con un intervalo de 7 dias entre las dos aplicaciones. Principales medidas de resultados: Determinación de la reproducibilidad del cuestionario. Resultados: En la determinación de la reproducibilidad, los valores de alfa-Cronbach fueron elevados, entre 0,97 y 0,98 para ambos sexos, y con un error técnico de medida entre 1,78\% y 3,10\%. El coeficiente de correlación intra-clase de Pearson fue entre 0,81 y 0,89, cuyos valores le otorgan al instrumento una fuerte capacidad de reproducibilidad. Conclusiones: El cuestionario desarrollado para valorar la actividad física de escolares de colegios públicos presenta valores aceptables de reproducibilidad y confiabilidad. Por lo tanto, los resultados sugieren su posible aplicación a poblaciones con similares características.
\end{abstract}

Palabras clave: Esfuerzo físico, ergometría, escolaridad, ejercicio, cuestionario.

Abstract

Background: So far there is no questionnaire to assess the level of physical activity related to children and adolescents' health in Peru, as well as information on measures of reproducibility. Objectives: To propose and validate a physical activity questionnaire for school adolescents living at moderate altitude. Design: Descriptive simple study. Setting: Faculty of Physical Education, UNICAMP, Brazil. Participants: School children 10 to 18 year-old. Interventions: The sample consisted of 116 students selected from a stratified probability, 58 men and 59 women, constituting 20\% of National College of Immaculate Conception's population, school located at District Jose Luis Bustamante y Rivero Arequipa (Peru). The questionnaire had 11 questions that assessed the level of physical activity using four indicators including such as activity type, frequency, duration and intensity. To determine reproducibility, test and re test procedure were done with an interval of seven days. Main outcome measures: To determine reproducibility of the questionnaire. Results: Cronbach's alpha to determine reproducibility showed high values between 0.97 and 0.98 for both sexes and a technical measurement error between 1.78 and 3.10\%. Intra-class Pearson's correlation coefficient was between 0.81 and 0.89 , values that give the instrument strong reproducibility. Conclusions: The questionnaire developed to assess physical activity of public schools children had acceptable levels of validity and reliability. Results suggest its possible application to populations with similar characteristics.

Key words: Exertion, ergometry, educational/status, excercise, questionnaires.

An Fac med. 2012;73(4):307-13 


\section{INTRODUCCIÓN}

El ejercicio físico y la aptitud física en niños y adolescentes se han tornado objeto de estudio entre los investigadores del área de las ciencias del movimiento humano, con una fuerte contribución al estudio del crecimiento, desarrollo y aptitud física relacionada a la salud. En este sentido, según Pitanga ${ }^{(1)}$, el ser humano se ha ido convirtiendo cada vez menos dependiente de sus capacidades físicas para sobrevivir, esto debido a que en los tiempo modernos se han reducido y en algunos casos se han extinguido las actividades laborales y cotidianas con predominancia física. En consecuencia, los hábitos de los niños y adolescentes son menos activos; si por un lado los recursos tecnológicos han aumentando la productividad de los bienes de consumo, por otro han contribuido negativamente sobre los niveles basales de aptitud física ${ }^{(2)}$.

Desde esa perspectiva, la aptitud física definida como la capacidad de ejecutar actividades físicas con energía y vigor sin exceso de fatiga, ha conducido a un menor riesgo de desarrollar enfermedades e incapacidades funcionales ${ }^{(3)}$. En ese sentido, la importancia de la evaluación de la aptitud física en escolares se refleja en el hecho de que los valores de los componentes morfológicos y funcionales de aptitud física por encima de la media poblacional, indican una menor exposición a riesgos de varias enfermedades crónicodegenerativas, tales como, cáncer, dislipidemia, hipertensión, diabetes y obesidad (4). De esta forma, un índice bajo de condición física es considerado como un fuerte predictor de enfermedades cardiovasculares, no solo en escolares con sobrepeso u obesidad, sino también en escolares eutróficos ${ }^{(5)}$. Cabe resaltar que los estudios en niños que relacionan la condición física con la salud no son demasiados, pero existen datos de países desarrollados que demuestran que, en niños y niñas de 13 a 18 años, un grado bajo de condición física se relaciona de forma direc- ta con un riesgo aumentado de padecer enfermedades cardiovasculares en edades avanzadas ${ }^{(6,7)}$.

Así mismo, existe una fuerte asociación entre la práctica de actividad física y un mejor patrón de salud, que ha sido citado en la literatura hace mucho tiempo y ha aumentado en la década actual ${ }^{(8-11)}$, por lo que se considera que una reducción en el nivel de actividad física realizada por los niños, ha provocado un descenso en el estado de la condición física, con la consiguiente influencia sobre la salud y calidad de vida ${ }^{(12-15)}$.

Por otro lado, debido a la complejidad y subjetividad que la actividad física representa, existen más de 50 técnicas diferentes para evaluarla. Dentro de los métodos indirectos, los cuestionarios han sido los más utilizados para evaluar la actividad física y el gasto energético, debido principalmente al costo financiero bajo y la baja demanda de tiempo baja para su aplicación ${ }^{(16)}$. El uso de los cuestionarios proporciona un buen nivel de detalle, permitiendo a los investigadores estudiar tanto la actividad física como los hábitos sedentarios, destacándose ya desde la década de 1960; su aplicación ha sido representativa para ayudar al estudio de las tendencias nacionales en el comportamiento de la actividad física y sedentarismo, en los últimos cinco años, para una población en muchos países, incluyendo Australia, Canadá, Estados Unidos y países de América Latina ${ }^{(17)}$.

Finalmente, teniendo en cuenta estas ventajas, en algunos países, como el caso del Perú y específicamente para poblaciones de moderada altitud, no existe un instrumento para la evaluación del nivel de actividad física. Por lo que, el objetivo del presente estudio fue proponer y validar un cuestionario de actividad física para escolares adolescentes de moderada altitud.

\section{MÉTODOS}

El estudio es descriptivo de corte transversal, teniendo como objetivo prin- cipal la validación de un instrumento cualitativo para una población de adolescentes de Escuelas públicas de la Ciudad de Arequipa, Perú.

De un total de 586 escolares, se seleccionó de forma probabilística estratificada 116 alumnos, siendo 58 varones y 59 damas. La muestra finalmente quedó constituida por el $20 \%$ del universo total (tabla 1).

En todos los casos, los escolares pertenecían al Colegio Nacional Inmaculada Concepción, el cual está localizado en la Jurisdicción de la Unidad de Gestión Educativa de la Provincia de Arequipa, Zona Sur. La provincia de Arequipa está ubicada a $2320 \mathrm{msnm}$. El Colegio está localizado en la zona urbana del Distrito de José Luis Bustamante y Rivero, donde, por lo general en el Perú, los niños y adolescentes que frecuentan las escuelas de zonas urbano-marginales son de condición socioeconómica baja y los que viven en zonas rurales de altitud son de condición muy baja.

El intervalo de edades comprende, en el caso de las niñas, desde los 11 hasta los 17 años y en los varones desde los 10 hasta los 18 años. Todos los escolares al momento de la aplicación del instrumento realizaban por lo general como parte de las sesiones de Educación Física 90 minutos de contenidos de actividades físicas, los que según el sistema educativo peruano deben ser realizados una vez por semana.

El instrumento elaborado cuenta con 11 cuestiones que evalúan el nivel de actividad física que desarrollan por lo general los adolescentes de las escuelas públicas de la provincia de Arequipa, Perú.

Para la construcción del instrumento, se procedió a la operacionalización de la variable 'actividad física', para lo cual se desagregó en indicadores y subindicadores (validez de contenido). Las preguntas fueron de tipo cerradas, en el que se incluyó alternativas múltiples, permitiendo marcar una sola al- 
Tabla 1. Operacionalización de la variable 'actividad física'.

\begin{tabular}{|c|c|c|}
\hline Variable & Indicador & Sub-indicadores/preguntas \\
\hline \multirow{15}{*}{ Actividad física } & \multirow{5}{*}{ Tipo de actividad física } & Deportiva \\
\hline & & Laboral (mecánica, agrícola) \\
\hline & & Recreativa \\
\hline & & Artística \\
\hline & & Ninguna \\
\hline & \multirow{3}{*}{ Frecuencia } & Diaria \\
\hline & & Semanal \\
\hline & & Mensual \\
\hline & \multirow{3}{*}{ Duración } & Menos de 10 minutos \\
\hline & & Entre 10 y 30 minutos \\
\hline & & Más de 30 minutos \\
\hline & \multirow{4}{*}{ Intensidad } & Vigorosa \\
\hline & & Moderada \\
\hline & & Leve \\
\hline & & Baja \\
\hline
\end{tabular}

ternativa para cada pregunta. Dicho procedimiento puede ser observado en la tabla 1.

Para validar el instrumento se optó por dos tipos: validez de contenido y validez de constructo intra-pruebas, como sugieren varios autores ${ }^{(18-20)}$.

Para la validez de contenido se procedió a operacionalizar la variable 'actividad física', como se describe en la tabla 1. Este tipo evalúa la correspondencia del instrumento con su contexto teórico y la congruencia de las preguntas con sus respectivos indicadores.

Para demostrar la validez del constructo, con base en la teoría o esquema teórico elaborado a partir de la operacionalización de la variable, se utilizó el coeficiente de Cronbach ${ }^{(21)}$, basado en la varianza de los ítems del instrumento.

Para establecer la confiabilidad del instrumento, se usó el coeficiente de confiabilidad de estabilidad, por medio de la fórmula de Spearman-Brown y Guttman ${ }^{(22)}$. Para este procedimiento, se aplicó el instrumento en dos oportunidades, teniendo un intervalo de una semana entre cada medición.

\section{Procedimientos para recolección de los datos}

Todos los alumnos fueron informados sobre la finalidad del estudio, así como se describió y explicó sobre el procedimiento del llenado del instrumento. Todo el procedimiento fue realizado por un mismo evaluador. Se administró el cuestionario en las aulas de las instalaciones del Colegio Nacional Inmaculada Concepción, según el horario de estudio $(7,30 \mathrm{~h}$ a $12,30 \mathrm{~h}$ y de $13,00 \mathrm{~h}$ a $18,00 \mathrm{~h}$ ). Todos los alumnos disponían de 30 minutos para llenar el respectivo cuestionario, así como fueron aprovisionados del respectivo material necesario para el llenado del instrumento.

El estudio contó con la respectiva aprobación del comité de ética del departamento médico del Instituto del Deporte Universitario de la Universidad Nacional de San Agustín de Arequipa (Perú), así como los padres y tutores de los niños llenaron y firmaron la ficha de consentimiento para autorizar la aplicación de la encuesta.

Los resultados fueron procesados por medio de un programa estadístico Sigma Estat $(7,0)$, a través del cual se utilizó la estadística descriptiva de media aritmética $(\mathrm{X})$, desviación estándar $(\mathrm{DE})$ y varianza $\left(\mathrm{S}^{2}\right)$.

Por otro lado, para validar se utilizó la fórmula de Cronbach, para la confiabilidad el error técnica de medida (ETM), y para la reproducibilidad el coeficiente de Correlación productomomento de Pearson. A su vez, para determinar las diferencias entre las medias y los límites extremos de concordancia, se utilizó la técnica de plotage, propuesto por Bland y Altman ${ }^{(23)}$.

\section{RESULTADOS}

Las tablas 2 y 3 muestran las características generales de la muestra estudiada, donde se observa el rango de edad de los adolescentes estudiados (11 a 17 años) para ambos géneros. Los es-

Tabla 2. Selección de la muestra.

\begin{tabular}{|c|c|c|c|c|c|c|}
\hline \multirow{2}{*}{ Edad (años) } & \multicolumn{2}{|c|}{ Mujeres } & \multicolumn{2}{|c|}{ Hombres } & \multicolumn{2}{|c|}{ Total } \\
\hline & Población & Muestra & Población & Muestra & Población & Muestra \\
\hline 11 & 60 & 12,0 & 60 & 12,0 & 120 & 24,0 \\
\hline 12 & 57 & 11,4 & 58 & 11,6 & 115 & 23,0 \\
\hline 13 & 62 & 12,4 & 63 & 12,6 & 125 & 25,0 \\
\hline 14 & 55 & 11,0 & 55 & 11,0 & 110 & 22,0 \\
\hline 15 & 58 & 11,6 & 58 & 11,6 & 116 & 23,2 \\
\hline 16 & 62 & 12,4 & 63 & 12,6 & 125 & 25,0 \\
\hline 17 & 55 & 11,0 & 55 & 11,0 & 110 & 22,0 \\
\hline Total & 409 & 81,8 & 412 & 82,4 & 821 & 164,2 \\
\hline
\end{tabular}


Tabla 3. Caracterización de la muestra.

\begin{tabular}{cccccc} 
& Edad (años) & Edad mínima & Edad máxima & $\begin{array}{c}\text { Horas/aula de } \\
\text { estudio semanal } \\
\text { (min) }\end{array}$ & $\begin{array}{c}\text { Horas/aula de } \\
\text { educación física } \\
\text { semanal (min) }\end{array}$ \\
Damas & $13,2 \pm 2,21$ & 11,0 & 17,0 & 1575 & 90 \\
Varones & $13,0 \pm 1,96$ & 10,0 & 18,0 & 1575 & 90 \\
Ambos & $13,1 \pm 2,06$ & 10,0 & 18,0 & 1575 & 90 \\
\hline
\end{tabular}

Tabla 4. Valores del coeficiente de Cronbach (alfa) para validar el cuestionario de actividad física.

\begin{tabular}{|c|c|c|c|c|c|c|}
\hline & \multicolumn{2}{|c|}{ Varones } & \multicolumn{2}{|c|}{ Damas } & \multicolumn{2}{|c|}{ Ambos géneros } \\
\hline & Evaluación 1 & Evaluación 2 & Evaluación 1 & Evaluación 2 & Evaluación 1 & Evaluación 2 \\
\hline N & 26 & 15 & 32 & 13 & 58 & 28 \\
\hline Cronbach & 0,98 & 0,94 & 0,97 & 0,92 & 0,97 & 0,98 \\
\hline
\end{tabular}

colares, en el momento en que fueron encuestados, realizaban un ciclo de estudios que comprende 45 minutos, la hora pedagógica dentro del proceso de enseñanza-aprendizaje, correspondiendo un total de 1575 minutos por semana, 315 minutos por día (45/aula). A su vez, realizaban las clases de educación física una vez por semana durante 90 minutos.

Los resultados mostrados en la tabla 4 permiten destacar los valores obtenidos para validar el cuestionario de actividad física por medio de la validez de constructo intra-pruebas. Para lo cual, se utilizó el coeficiente de alfa Cronbach, encontrándose estabilidad entre los ítems que evalúan los indicadores del cuestionario.

Los valores obtenidos de alfa Cronbach por género oscilaron para la primera evaluación entre 0,97 a 0,98 y para segunda evaluación entre 0,92 a 0,94 y para ambos géneros entre 0,97 a 0,98 . Por lo tanto, destacamos que los valores de validez, tanto de forma individual para cada género y de forma mixta para ambos géneros, muestran valores altos de validez, y por ende de predicción.

La variabilidad de las medidas es considerada como importante indicador de la capacidad de medición de las variables. En este caso particular, la estabilidad del cuestionario aplicado muestra valores aceptables de error técnico de medida (ver tabla 5) oscilando entre 1,78 y $3,10 \%$, siendo inferiores al $5 \%$, comúnmente utilizado como un limite máximo de error permisible. Por lo tanto, la encuesta aplicada se muestra altamente confiable para este tipo de población.

En cuanto a la capacidad de reproducibilidad, el coeficiente de Pearson muestra valores de $(r)$ significativos, oscilando los valores entre 0,81 a 0,89. Por lo que la capacidad de reproducibilidad de la encuesta es alta. Esto sugiere que el instrumento elaborado para adolescentes de centros educativos públicos puede ser aplicado a poblaciones con características similares, puesto que cumple con los requisitos de fiabilidad necesarios. Las figuras 1,2 y 3 ilustran la concordancia de las medias obtenidas y los límites extremos $( \pm 2 \mathrm{DE})$ entre el test y re-test de la encuesta que evalúa la actividad física de escolares.

\section{DISCUSIÓN}

La monitorización de los niveles de práctica habitual de actividad física en poblaciones jóvenes se ha convertido en un tema de interés y preocupación constante entre los especialistas del área ${ }^{(24)}$. Fruto de ello es que, en los últimos 15 a 20 años, los avances tecno- lógicos y metodológicos han facilitado enormemente la medición del gasto energético humano, tanto en laboratorio como en el campo ${ }^{(25)}$, surgiendo métodos volcados a la evaluación de la práctica de la actividad física, como por ejemplo, agua doblemente marcada, observación directa, censores de movimiento, frecuencia cardiaca y los registros de auto-recordatorio ${ }^{(26)}$.

Desde esa perspectiva, con el objetivo de desarrollar un cuestionario de actividad física para adolescentes de escuelas públicas, el estudio tomó en consideración las sugerencias descritas por Dishman, Sunhard ${ }^{(27)}$, al destacar los requisitos mínimos que un instrumento de medición debe presentar, como son: la validez y confiabilidad. A ese respecto, los resultados evidencian valores altos de validez, puesto que el coeficiente de alfa Cronbach muestra valores de consistencia entre 0,92 a 0,98 , tanto en la primera, como en la segunda evaluación. Estos valores son superiores en relación a otros estudios cuando evaluaron las estrategias motivacionales en las clases de educación física ${ }^{(28,29)}$, así como instrumentos que miden el clima motivacional en el deporte ${ }^{(30,31)}$ y las creencias sobre las causas del éxito en el deporte ${ }^{(32)}$. En este sentido, los resultados obtenidos en cuanto a la validez de constructo realizados por medio del coeficiente de alfa de Cronbach suponen comprobar la adecuación teórica del mismo a través del establecimiento de relaciones con otros constructos teóricos. Por lo tanto, se deduce que el instrumento desarrollado muestra validez para adolescentes que frecuentan centros escolares públicos que realizan por lo general una sesión de 90 minutos de actividad física

Tabla 5. Confiabilidad y reproducibilidad del cuestionario de actividad física.

\begin{tabular}{ccc} 
& ETM & Pearson \\
Varones & 2,40 & $0,82^{*}$ \\
Damas & 3,10 & $0,89^{*}$ \\
Ambos géneros & 1,78 & $0,81^{*}$ \\
\hline
\end{tabular}

ETM = error técnico de medida, ${ }^{*}=p<0,001$. 


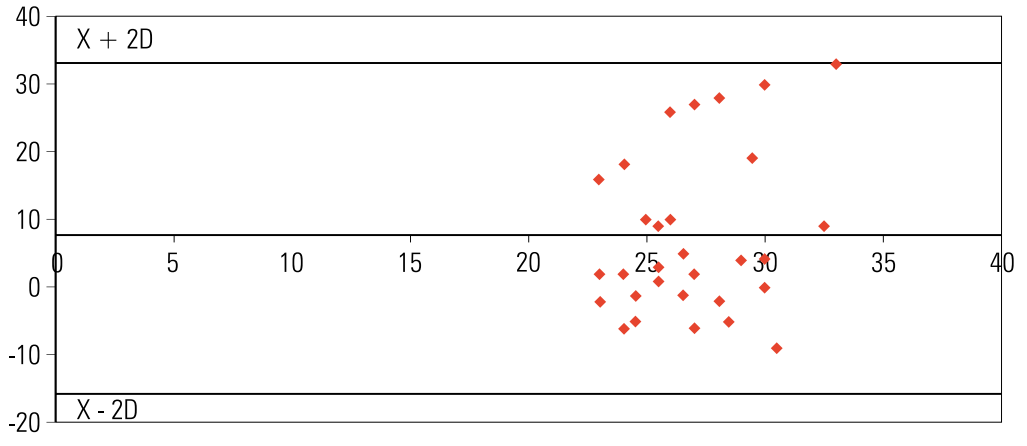

Figura 1. Concordancia de las medias entre el test y re-test para escolares de ambos géneros, según el plotaje de Bland y Altman.

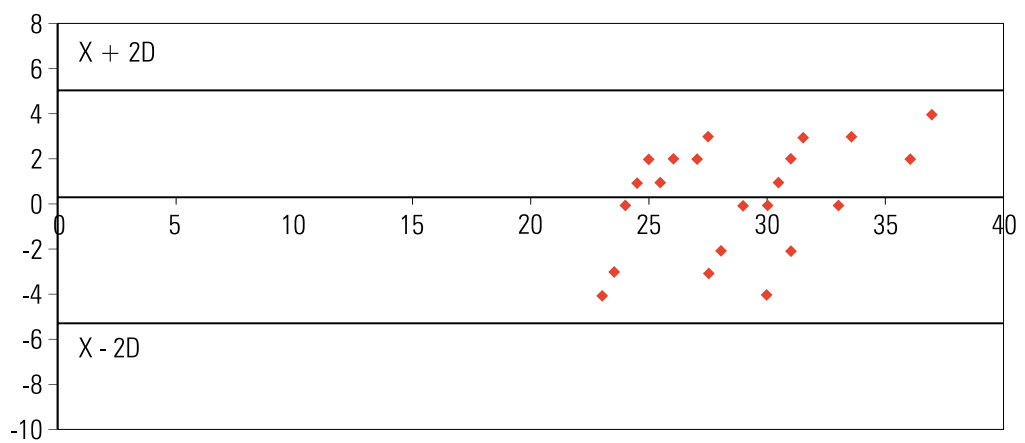

Figura 2. Concordancia de las medias entre el test y re-test para varones, según el plotaje de Bland y Altman.



Figura 3. Concordancia de las medias entre el test y re-test para damas, según el plotaje de Bland y Altman. durante una semana. Inclusive, recientes investigaciones sugieren la posibilidad de usar encuestas para evaluar el uso del tiempo, para la vigilancia de la actividad física y el comportamiento de la población sedentaria ${ }^{(33,34)}$, así como para estudios de naturaleza epidemiológica ${ }^{(24)}$, puesto que entre sus ventajas podemos mencionar su costo bajo y rapidez, ya que no requieren del seguimiento de los sujetos de estudio ${ }^{(35)}$.

Respecto a la confiabilidad de las medidas, nos basamos en la técnica del test re-test, cuyo intervalo de tiempo fue de siete días entre cada medición, para lo cual utilizamos el coeficiente de correlación intra-clase de Pearson, error técnico de medida (ETM) y el plotaje de Bland, Altman ${ }^{(23)}$. Los resultados del estudio muestran similares coeficientes de correlación con otros estudios que investigaron varios tipos de cuestionarios en adolescentes ${ }^{(36-38)}$. Esto evidencia que el cuestionario propuesto muestra capacidad alta de reproducibilidad de los resultados, manteniendo estabilidad entre las mediciones. Esto puede observarse en el plotaje de Bland, Altman, donde el intervalo de concordancia de las medias obtenidas se encuentra dentro los límites extremos $( \pm 2 \mathrm{DE})$ entre el test y re-test de la encuesta. A su vez, el ETM es inferior a los límites permisibles expresados en la literatura en 5\%, oscilando los valores del estudio entre 1,78 a $3,10 \%$, respectivamente.

En conclusión, nuestro medio carece de estudios que engloben encuestas transversales que midan los niveles de actividad física de poblaciones escolares y adultas. Los estudios sugieren que es necesario estudiar la prevalencia, seguimiento y la tendencia de las poblaciones por medio de cuestionarios ${ }^{(17,39)}$. Esto, en razón de que el sedentarismo $\mathrm{y}$ otros factores, fundamentalmente los malos hábitos alimenticios, han contribuido al cambio experimentado en los últimos decenios ${ }^{(13)}$, no solo en países desarrollados o en vías de desarrollo, sino también en países subdesarrollados. Por lo tanto, el cuestionario propuesto para adolescentes de ambos 
sexos que frecuentan escuelas públicas cumple con los requisitos de validez y confiabilidad y podría ser generalizado a poblaciones con características similares, a pesar de que en el estudio no se controló algunas variables como la condición socio-económica y el estado nutricional, los que de cualquier forma hubieran permitido una mejor discusión de los resultados obtenidos. Por lo tan- to, cualquier generalización en relación al cuestionario de actividad física para escolares adolescentes de moderada altitud debe ser analizada con cautela y precaución.

\section{CUESTIONARIO DE NIVEL DE ACTIVIDAD FÍSICA}

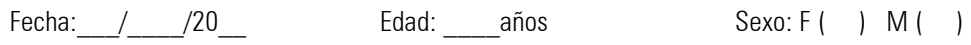

Nosotros estamos interesados en conocer qué nivel de actividad física los adolescentes realizan como parte de su día a día. Sus respuestas nos ayudarán a entender qué tan activos son los adolescentes. Las preguntas están relacionadas al tiempo que tú ocupas realizando actividad física en una semana Las preguntas incluyen las actividades que tú realizas en el colegio, para ir de un lugar a otro, por realizar, por deporte, por ejercicio o como parte de tus actividades en casa 0 en un club, academia.

Sus respuestas son MUY IMPORTANTES. Por favor responde cada pregunta. Gracias por tu participación.

Para responder las preguntas recuerda que:

Actividades físicas VIGOROSAS son aquellas que necesitan de un gran esfuerzo físico y que te hacen respirar MUCHO más fuerte que lo normal. Las actividades físicas MODERADAS son aquellas que necesitan de algún esfuerzo físico y que te hacen respirar UN POCO más fuerte que lo normal.

1. ¿Realizas tú algún tipo de actividad física por lo general durante la semana?

a) Un deporte en específico

b) Trabajar (mecánica, construcción, cosecha)

c) Algún tipo de ejercicio (caminata, paseo en bicicleta, juegos recreativos de carrera), tareas domésticas en casa (limpieza, lavandería)

d) Recreación pasiva (juego de mesa), tocar un instrumento, asistir a academias de idiomas

e) Ninguna

2. ¿Cómo acostumbras tú a ocupar tu tiempo libre (fuera del horario escolar)?

a) En ninguna actividad

b) Mirando televisión

c) Artes, pintura, trabajos manuales, academias de reforzamiento o idiomas d) Actividades de ejercicio moderadas como caminar en el parque, paseo en bicicleta

e) Deportes vigorosos como el fútbol, basket, o actividades en mecánica, construcción.

3. ¿Realizas tú alguna actividad física durante el receso (recreo escolar)?

a) Práctica de deportes como fútbol, voley, basket

b) Juegos recreativos (pesca, escondidas)

c) Actividades como pintura, música, costura

d) Ninguna actividad

4. En los meses de vacaciones, ¿tú realizas actividad física?

a) Ninguna

b) Solo a veces

c) A menudo

d) Siempre

5. ¿Cuántos días por semana tú realizas actividad física?

a) Ninguno

b) 203

c) 405

d) Más de 5
6. En los días que tú realizas actividad física icuanto tiempo (minutos) total ocupas por día?

a) Menos de 10 minutos

b) De 10 a 30 minutos

c) De 30 a 40 minutos

d) Más de 40 minutos

7. ¿Cuántos días/semana tú realizas actividad física vigorosa?

a) Ninguna

b) 203

c) 405

d) Más de 5

8. ¿Cuántos minutos/día tú realizas actividad física vigorosa?

a) Menos de 10 minutos

b) De 10 a 30 minutos

c) De 30 a 40 minutos

d) Mas de 40 minutos

9. ¿Cuántos días/semana tú realizas actividad física moderada?

a) Ninguna

b) 203

c) 405

d) Más de 5

10 ¿Cuántos minutos/día tú realizas actividad física moderada?

a) Menos de 10 minutos

b) De 10 a 30 minutos

c) De 30 a 40 minutos

d) Más de 40 minutos

11 ¿Tú acostumbras ir de la casa al colegio en?

a) Movilidad particular

b) Transporte urbano

c) A pie 


\section{REFERENCIAS BIBLIOGRÁFICAS}

1. Pitanga, FJG. Epidemiología da Atividade Física. Rev Bras Cien Mov. 2002;10(3):49-54.

2. Silva de Araujo, S; Cabral de Oliveira, A. Aptidão física em escolares de Aracaju. Rev Bras Cineantropom Desempenho Hum. 2008;10(3):271-6.

3. Silva RJS, Silva Junior AG, Cabral de Oliveira AC. Crescimento em crianças e adolescentes: un estudo comparativo. Rev Bras Cineantropom Desempenho Hum. 2005;7(2):55-61.

4. Guedes DP, Guedes JERP, Barbosa DS, Oliveira, JA. Atividade física habitual e aptidão física relacionada à saude em adolescentes. Rev Bras Cienc Mov. 2002;10(1):13-21.

5. Wei M, Kampert JB, Barlow CE, Nichaman MZ, Gibbons LW, Paffenbarger RS, et al. Relationship between low cardiorespiratory fitness and mortality in normal-weight, owerweight, and obese men. JAMA. 1999;282:1547-53.

6. Ortega FB. Ruiz JR. Castillo MJ. Moreno LA. González-Gross M. Warnberg J. Bajo nivel de forma física en los adolescentes españoles. Importancia para la salud cardiovascular futura (Estudio AVENA). Rev Esp Cardiol. 2005;58:898-909.

7. González-Gross M. Ruiz JR. Moreno LA. De Rufino-Rivas P. Garauler M, Mesana MI, et al. Body composition and physical performance of Spanish adolescents: the AVENA pilot study. Acta Diabetol. 2003;40 suppl 1:S299-301.

8. Skinner JS, Benson H, Mcdonough JR, Hames CG. Social status, physical activity, and coronary proneness. J Chron dism 1986;19:773-83.

9. Caspersen CJ, Bloemberg BMP, Saris WHM, Merrit RK, Kromhout D. The prevalence of select physical activities and their relation with coronary heart disease risk factors in elderly men: The Zutphen Study, 1985. Am J Epidemiol. 1991;133:1078-92.

10. Gordon DJ, Witztum JL, Hunninghake D, Gates S, Glueck CJ. Habitual physical activity and highdensity lipoproteina colesterol in men with primary hipercolesterolemia. Circulation. 1983;67:512-20.

11. Matsudo VKR, Matsudo SMM. Evidencias da importancia da atividade física nas doenças cardiovasculares e na saúde. Diagnóstico \& Tratamento. 2000;5(2):10-7.

12. Guerra S, Duarte J, Mota J. Physical activity and cardiovascular disease risk factors in shoolchildren. Eur Phys de Rev. 2001;7(3):269-81.

13. Pierón M. Estilo de vida, práctica de actividades físicas y deportivas, calidad de vida. Actas de ponencias del II congreso Mundial de ciencias de la actividad Física y el deporte. Deporte y calidad de vida. Granada (España), 2003.

14. Ribeiro J, Guerra S, Pinto A, Oliveira J, Duarte J, Mota J. Overweight and obesity in children and adolecents: relationchip with blood pressure, and physical activity. Ann Hum Biol. 2002;30(2):203-13.
15. Salbe AD, Weyer C, Harper I, Lindsay RS, Ravussin E, Tataranni PA. Assessing the risk factors for obesity between chilhood and adolescents. II. Energy metabolism and physical activity. Pediatrics. 2002;110:307-14

16. Maluf F, Almeida M, Marques $P$, Bertoldo $T$. Questionários em medidas de atividade física em idosos. Rev Bras Cienatropom Desempenho Hum 2006;8(4):99-106.

17. Van der Ploeg HP, Merom D, Chau J, Bittman M Trost S, Bauman A. Advances in population surveillance for physical activity and sedentary behavior: reliability and validity of time use surveys. Am J Epidemiol Advance. 2010;172(10):1199-206.

18. Cronbach LJ, Meehl PE. Construct validity in psychological tests. Psychol Bull. 1955;52:281-302.

19. Kerlinger FN. Investigación del comportamiento: técnicas y metodología. México, DF: Nueva editorial Interamericana. Actualmente se publica por McGraw-Hill Interamericana, 1975.

20. American Psychological Association. APA Committee on ethical standard in psychological Research. Washington, 1974:172.

21. Cronbach LJ. Essentials of psychological testing. Nueva York: Gardner Press, 1984.

22. Guttman. Fundamentos del análisis del escalograma. En: Wainerman $\mathrm{CH}$ (comp). Escalas de medición en ciencias sociales. Buenos Aires. Ediciones Nueva Visión, 1976;291-330.

23. Bland JM, Altman DG. Statistical methods for assessing agreement between two methods for clinical measurement. Lancet. 1986;8:307-10.

24. Guedes DP, Lopes CC, Guedes JERP. Reprodutibilidade e validade do Questionário Internacional de Atividade Física em adolescentes. Rev Bras Med Esporte. 2005;11(2):151-8.

25. Leonard WR. Measuring human energy expenditure and metabolic function basic principles and methodos. J Anthropol Sci. 2010;88:221-30.

26. Montoye HJ, Kemper HCG, Saris WHM, Washburn RA. Measuring physical activity and energy expenditure. Champaign, Illinois: Human Kinetics, 1996.

27. Dishman R, Sunhard M. Reliability and concurrent validity for a 7-d re-call of physical activity in college students. Med Sci Sports Exercise. 1988;20(1):14-25.

28. Nunnally JC. Psychometric theory. New York McGraw-Hill, 1978.

29. Cervelló E, Moreno JA, del Villar F, Reina R. Desarrollo y validación de un instrumento de medida de las estrategias motivacionales empleadas en las clases de educación física. Rev Iberoam Psico Ejercicio Deporte. 2007;2(2):53-72.

30. Balaguer I, Guivernau M, Duda JL, Crespo M. Análisis de la validez de constructo y de la validez predictiva del cuestionario de clima motivacional percibido en el deporte (PMCSQ-2) con tenistas españoles de competición. Rev Psicol Deporte. 1997;11:41-57.

31. Cervelló EM, Escartí A, Balagué G. Relaciones entre la orientación de metas disposicional y la satisfacción con los resultados deportivos, las creencias sobre las cusas de éxito en deporte y la diversión con la práctica deportiva. Rev Psicol Deporte. 1999;8:7-19.

32. Duda JL, Fox KR, Biddle SJH, Amstrong N. Children's achievement goals and beliefs about success in sport. Brit J Educat Psychol. 1992;62:313-23

33. Tudor-Locke C, Bittman M, Merom D, et al. Patterns of walking for transport and exercise: a novel application of time use data. Int J Behav Nutr Phys Act. 2005;2:5.

34. Tudor-Locke C, Van der Ploeg HP, Bowles HR, et al. Walking behaviours from the 1965-2003 American Heritage Time Use Study (AHTUS). Int J Behav Nutr Phys Act. 2007;4:45.

35. Hernández B, Velasco-Mondragón HE. Encuestas transversales. Salud Publica México. 2000;42(5):447-55.

36. Aaron DJ, Kriska AM, Dearwater SR, Cauley JA, Metz KF, LaPorte RE. Reproducibility and validity of an epidemiologic questionnaire to assess past year physical activity in adolescents. Am J Epidemiol. 1995;142:191-201

37. Sallis JF, Buono MJ, Roby JJ. Seven-day recall and other physical activity self-report in children and adolescents. Med Sci Sports Exerc. 1993;25:99-108.

38. Evenson KR, McGinn AP. Test-retest reliability of a questionnaire to assess physical environmental factors pertaining to physical activity. Int J Behav Nutr Phys Act. 2005;2:7.

39. Trost SG, Mciver KL, Pate RR. Conducting accelerometerbased activity assessments in field-based research. Med Sci Sports Exerc. 2005;37(11 Suppl):S531-S543.

Artículo recibido el 25 de enero de 2012 y aceptado para publicación el 25 de junio de 2012.

Institución donde se realizó el trabajo:

Universidad Estadual de Campinas, Brasil

Correspondencia:

Rossana Gómez Campos

Av. Erico Verissimo 701

Cidade Universitaria, CEP, 13083-851

Campinas, SP: Brasil.

Correo-e: rossanagomez_c@hotmail.com 